A VMAT planning solution for prostate patients using a commercial treatment planning system

This article has been downloaded from IOPscience. Please scroll down to see the full text article.

2010 Phys. Med. Biol. 55 N395

(http://iopscience.iop.org/0031-9155/55/14/N01)

View the table of contents for this issue, or go to the journal homepage for more

Download details:

IP Address: 130.88.240.163

The article was downloaded on 02/07/2012 at 15:29

Please note that terms and conditions apply. 
NOTE

\title{
A VMAT planning solution for prostate patients using a commercial treatment planning system
}

\author{
C J Boylan, C Golby and C G Rowbottom \\ North Western Medical Physics, The Christie NHS Foundation Trust, Wilmslow Road, \\ Manchester M20 4BX, UK \\ E-mail: Christopher.Boylan@physics.cr.man.ac.uk
}

Received 23 December 2009, in final form 15 June 2010

Published 5 July 2010

Online at stacks.iop.org/PMB/55/N395

\begin{abstract}
Volumetric modulated arc therapy (VMAT) is a rotational delivery technique which offers the potential of improved dose distributions and shorter treatment times when compared to fixed-beam intensity-modulated radiation therapy (IMRT). This note describes the use of an existing treatment planning system (Philips Pinnacle ${ }^{3}$ v.8.0), supplemented by in-house software, to produce a single-arc VMAT prostate plan. While a number of planning systems for the Elekta VMAT platform are commercially available, the use of an in-house solution has allowed more detailed investigations of VMAT planning, as well as greater control over the optimization process. The solution presented here begins with a static step-and-shoot IMRT approach to provide initial segment shapes, which are then modified and sequenced into 60 equally spaced control points in a $360^{\circ}$ arc. Dose-volume histogram comparisons demonstrate that this VMAT planning method offers multiple dose level target coverage comparable to that from a standard IMRT approach. The VMAT plans also show superior sparing of critical structures such as the rectum and bladder. Delivery times are reduced with the VMAT method, and the results of dosimetric verification, resilience and repeatability tests indicate that the solution is robust.
\end{abstract}

(Some figures in this article are in colour only in the electronic version)

\section{Introduction}

Volumetric modulated arc therapy (VMAT) offers the potential to deliver intensity-modulated dose distributions comparable to or better than those produced by conventional intensitymodulated radiation therapy (IMRT) treatments, in a shorter delivery time (Palma et al 2008, Shaffer et al 2010, Rao et al 2010). As described by Otto (2008), the technique involves varying a linear accelerator's dose rate, field shape and gantry speed in an arc to produce the desired dose distribution. The Elekta VMAT solution (Elekta Ltd, Crawley, UK) which 
is considered in this note has been successfully commissioned by this centre and has been found by others to be a robust method for delivering conformal radiotherapy (Bedford and Warrington 2009).

In IMRT treatment planning, inverse-planned solutions are commonplace. However, VMAT provides a more complex problem for the optimization algorithm. There are a large number of parameters to be optimized, and a variety of machine limitations which must be taken into account. The maximum leaf speed, jaw speed and gantry speed affect the efficiency of VMAT delivery and the dosimetric quality of the plans. Furthermore, the Elekta VMAT system chooses from a discrete set of dose rates in order to deliver the desired dose. For example, a nominal maximum dose rate of $600 \mathrm{MU} \mathrm{min}^{-1}$ yields dose rate bins of $600 \mathrm{MU}_{\mathrm{min}^{-1}}, 300 \mathrm{MU} \mathrm{min}^{-1}, 150 \mathrm{MU} \min ^{-1}$, $75 \mathrm{MU} \min ^{-1}, 37 \mathrm{MU} \min ^{-1}$ and $18 \mathrm{MU}$ $\min ^{-1}$. Ideally these constraints should be considered as part of the inverse optimization, such that the resultant plan is capable of being delivered accurately and efficiently.

There are a number of planning solutions available for Elekta VMAT, including the SmartArc module for the Pinnacle ${ }^{3}$ v.9.0 treatment planning system (Philips Medical Systems, WI, USA). SmartArc uses an optimization algorithm described by Bzdusek et al (2009), and although the clinical version of the software has only recently been released, it has been shown to produce VMAT plans of equivalent quality to IMRT for certain sites (Guckenberger et al 2009, Bertelsen et al 2010). Other VMAT planning methods have been proposed by Bedford (2009), Cao et al (2009) and Matuszak et al (2010) — all of which implement aspects of the earlier Pinnacle v.8.0 planning system.

While the initial results from SmartArc appear promising, 'in-house' planning methods currently allow for more detailed investigations of VMAT planning, providing more flexibility and control over the optimization process, and include the ability to adjust control point parameters (such as leaf positions and weighting) after the optimization process has finished (not currently possible with SmartArc).

This note describes the commissioning of a VMAT planning solution for prostate patients, using Pinnacle v.8.0 and software developed in-house. The solution, which delivers multiple dose level distributions comparable to IMRT, produces a single $360^{\circ}$ arc which can be delivered in a short time and to a high degree of dosimetric accuracy. Dynamic machine constraints are considered in the in-house software, such that the resultant plan is deliverable and efficient. Comparisons are made with step-and-shoot IMRT by evaluating dose-volume parameters and conformity to the Conventional or Hypofractionated High dose Intensity-modulated radiotherapy for Prostate cancer (CHHIP) trial protocol (Khoo and Dearnaley 2008). The results of dosimetric verification using a three-dimensional detector array are described, and the repeatability and resilience of delivery are investigated.

\section{Methods}

\subsection{Treatment planning process}

2.1.1. Plan setup and prescription. The treatment planning system used was Pinnacle ${ }^{3}$ v.8.0m, with the direct machine parameter optimization (DMPO) module for IMRT. Prostate patients were CT scanned in a supine position according to a standard protocol with $5 \mathrm{~mm}$ axial slice width. The organs at risk (OARs) were contoured (rectum, bladder, femoral heads, urethral bulb and bowel) and target volumes were expanded according to the CHHIP protocol. For the pre-clinical commissioning patients described in this note and the clinical VMAT patients, the dose prescribed was 57 Gy in 19 fractions (equivalent to group 3 of the CHHIP 
Table 1. Selection of some of the relevant dosimetric quality parameters outlined in the CHHIP trial protocol.

\begin{tabular}{lr}
\hline CHHIP trial parameter & Constraint $(\%)$ \\
\hline PTV1 min & 76 \\
PTV2 min & 91 \\
PTV3 min & 95 \\
PTV3 median & -101 \\
Bladder V68 max & 50 \\
Bladder V81 max & 25 \\
Bladder V100 max & 5 \\
Rectum V68 max & 60 \\
Rectum V81 max & 50 \\
Rectum V88 max & 30 \\
Rectum V95 max & 15 \\
Rectum V100 max & 3 \\
\hline
\end{tabular}

trial). The trial also specified three dose levels to three different planning target volumes (PTVs); these are detailed along with other specifications in table 1.

2.1.2. Initial optimization. 15 equi-spaced $8 \mathrm{MV}$ beams ( $24^{\circ}$ apart) were added to the plan starting at a gantry angle of $192^{\circ}$ and ending at $168^{\circ}$. A fixed collimator angle of $10^{\circ}$ was applied to avoid excessive inter-leaf leakage dose to the patient from the rotational delivery technique. DMPO was then used to create a 'step-and-shoot' plan, using a class solution of dose constraints derived from a standard IMRT solution. The optimization parameters were set such that the maximum number of control points was 26 (i.e. each beam contains 1 or 2 control points after optimization). The minimum segment area was set to $20 \mathrm{~cm}^{2}$ and segments with less than $6 \mathrm{MUs}$ were removed at this stage. Low-weighted segments can result in a poorer quality delivery, due to the inherent instability of the linear accelerator at low dose rates. The dose calculation was performed with a collapsed-cone convolution algorithm, using a dose grid resolution of $0.3 \mathrm{~cm}$. Over 25 iterations, DMPO produced control points similar to those shown in figure 1 .

2.1.3. External sequencing of control points. At this stage there were 15 equi-spaced beams each with one to two control points. A Java application was developed in-house which interrogated the Pinnacle file system and modified the plan, dividing each beam containing two control points into two separate beams containing one control point each. Optimizing 15 fluence maps with a coarse gantry angle spacing and then re-sequencing the control points into an arc reduced the computation time considerably compared to optimizing 26 fluence maps initially (this is a similar principle to the one employed by Bzdusek et al (2009)). The two new beams were shifted $6^{\circ}$ in either direction, such that the spacing was $12^{\circ}$ between the beams. The individual weighting of each control point, and hence the number of monitor units, was retained. For the beams that contained a single control point at the end of the DMPO optimization, the beam was split into two identical control points, shifted $6^{\circ}$ in either direction, and the half the MUs from the original control point were given to each new beam. At the end of this process the arc contained 30 equi-spaced beams, from gantry angle $186^{\circ}$ to $174^{\circ}$. 


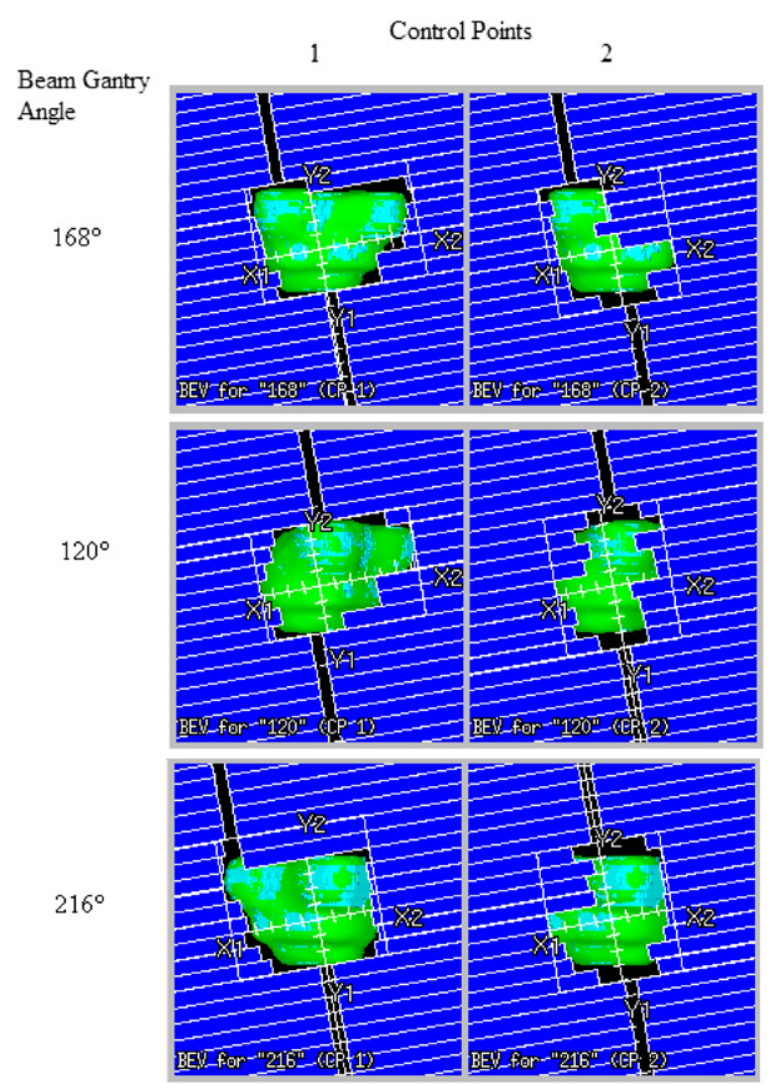

Figure 1. The two control points produced by DMPO for an example prostate patient for 3 of the 15 beams.

As there may be large differences in shape between each pair of control points, the amount of leaf motion between each of the newly created beams was minimized. In order to achieve this, the in-house software used a linear search (employing a 'greedy' algorithm) to determine the most efficient order of each pair of control points. The algorithm examined the leaf and jaw positions between adjacent control points, and ordered them such that leaf and jaw motion was minimized. In doing this, limitations of the VMAT delivery (i.e. maximum leaf and jaw travel per degree) were taken into account within the software to help to improve the efficiency of the resultant plan. The speed of delivery of a VMAT plan is determined by the dose rates selected by the linear accelerator, which is in turn determined by the difference between the positions of the leaves, jaws and gantry of adjacent control points. Minimizing leaf motion between the available control points ensures that the dose rate bins selected by the linac control system are as high as possible.

2.1.4. Interpolation of control points. Due to the continuous delivery of VMAT plans, initial testing indicated that the 30-beam step-and-shoot plan did not lead to a delivered dose distribution which matched the planning system prediction. Further investigation demonstrated that the coarse representation of 30 equi-spaced beams was not an adequate approximation to the continuous arc delivery employed by VMAT with a high degree of modulation between control points in the arc. Other authors have discussed the control point sampling required 

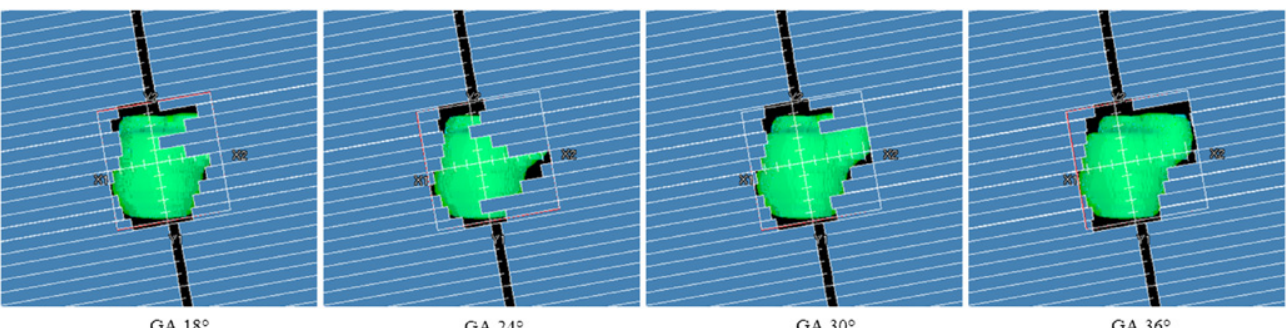

Figure 2. An example of four consecutive control points produced by the VMAT planning solution. Leaves outside of the field have been adjusted so that they remain stationary throughout the arc.

for accurate dosimetry (Otto 2008, Webb and McQuaid 2009, Feygelman et al 2010), and adequate results using 4 or $6^{\circ}$ spacings for prostate cases have been demonstrated (Bzdusek et al (2009) and Cao et al (2009), respectively). Therefore, a series of interpolated beams were added midway between each existing beam, taking an average of the leaf and jaw positions between adjacent control points. This was done again using the in-house software, and a plan with 60 equi-spaced beams with $6^{\circ}$ between each control point was produced. Monitor units were distributed such that half the MUs from the next control point in the arc were assigned to the interpolated beam.

2.1.5. Re-optimization and final dose calculation. After the control points were 'split' and interpolated, a further optimization step was required to refine the multi-leaf collimator (MLC) positions and beam weights and ensure that the dose distribution was clinically acceptable. This was achieved by running DMPO with the same parameters as the initial optimization, but using the 60 control points as the starting point for the optimization. The DMPO optimization process then made small changes to both the leaf positions and segment weights to minimize the overall cost function. The maximum number of iterations was set to ten and the final dose calculation was performed.

At this stage, steps were taken to ensure that the delivery was as efficient as possible. Due to the $10^{\circ}$ collimator twist, the superior-inferior (Y) jaws were inspected and altered if the high dose region extended outside of the PTV. Following the final re-optimization and dose calculation it was found that leaves outside of the treatment field were required to move a significant amount between control points. This unnecessary motion increased the delivery time, as the gantry speed and dose rate had to be reduced to wait for the out-offield leaf motion to finish. It was also found that these plans resulted in poorer dosimetric verification results. Therefore, leaf motion outside of the field was minimized on Pinnacle prior to export and delivery. Figure 2 shows an example of four adjacent control points after sequencing.

The approved plans were exported in DicomRT plan format to a record-and-verify system (MOSAIQ). MOSAIQ converts the exported files into RTP format, which are simple text files containing the plan details including the beam parameters and monitor units. At this stage, the RTP file consisted of 60 discrete beams each containing one control point. In order for MOSAIQ and the linear accelerator control system to recognize and deliver the plan as a VMAT treatment, the RTP file had to be reformatted to contain one beam with 60 control points with the gantry angle changing in each. This was achieved with a further piece of software, Arc Converter (William Beaumont Hospital, MI, USA), which was modified and tested in-house. The plans were then imported into MOSAIQ and could be delivered as VMAT prescriptions. 


\subsection{Pre-clinical testing and verification}

Commissioning for the VMAT planning solution consisted of creating plans as described above on five randomly chosen prostate patients previously treated with IMRT. Dosevolume statistics were recorded for the IMRT and VMAT plans, along with CHHIP trial parameters.

Dosimetric verification was performed on the five patient plans using a three-dimensional detector array (Delta ${ }^{4}$, Scandidos, Sweden). The Delta ${ }^{4}$ has been demonstrated to be an appropriate device for the verification of VMAT treatments (Bedford et al 2009). Gamma analysis was performed at the $3 \% / 3 \mathrm{~mm}$ level, within the $20 \%$ isodose.

The resilience of delivery was also investigated using one of the five patient plans as a reference plan. Using the Delta ${ }^{4}$, the dosimetric effects of delivery under non-ideal scenarios were studied. These scenarios were: (a) interruption of the beam mid-treatment, (b) termination of the beam with completion on a partial beam, (c) simulated communication failure (i.e. manually disconnecting the MOSAIQ system from the linac control system midtreatment) with completion on a partial beam, (d) termination of the beam on a symmetry error (i.e. manually changing the beam symmetry mid-treatment) with completion on a partial beam and (e) deliveries separated by a time frame of greater than 3 months.

Finally, the effects of symmetry and flatness on dosimetric repeatability were investigated. The reference plan was delivered to the Delta ${ }^{4}$ with the symmetry of the treatment beam adjusted to be $\pm 5 \%$ in both the gun-target $(\mathrm{GT})$ and transverse (AB) directions. $5 \%$ asymmetry is an extreme test which lies beyond the clinical tolerance of the linear accelerator.

\section{Results}

\subsection{Comparison to IMRT plans}

A dose-volume histogram (DVH) comparison between the VMAT and IMRT plans for one of the commissioning patients is shown in figure 3. Target volume coverage (PTV1, PTV2 and PTV3) is equivalent for both techniques, demonstrating the ability of the VMAT solution to produce multiple dose level distributions. The VMAT plans offer superior avoidance of critical structures such as the rectum and bladder, which receive a lower volume of low to intermediate dose when compared to IMRT. The femoral heads receive a higher volume of low dose in the VMAT plan, although at around 15 Gy the histograms cross over and the VMAT plan is superior to the IMRT plan. In the example shown, dose to the bowel is higher in the VMAT plan, but at the CHHIP dose level of 38.76 Gy the absolute difference in irradiated volume between the VMAT and IMRT plans is $0.3 \mathrm{cc}$.

CHHIP parameters for all five patients are shown in figures 4(a)-(c). Again, target volume coverage is similar between the two techniques. OAR constraints are met by both techniques, with the VMAT plans performing better at the low to intermediate dose range for the rectum and bladder. The whole body volume receiving $20 \mathrm{~Gy}$ or more is lower for VMAT than for IMRT, indicating better conformality in the high dose region. As with many rotational delivery techniques, the volume of body receiving lower doses of $>5$ Gy and $>10$ Gy is higher for VMAT. However, the differences in low dose volumes between VMAT and IMRT are not significant; over the five patients the average V5 for the body (volume receiving 5 Gy or more) is $6558( \pm 825)$ cc for VMAT compared to $5977( \pm 632)$ cc for IMRT.

For the five patients examined as part of commissioning, the number of monitor units required for VMAT delivery is less than for step-and-shoot IMRT delivery (mean $521 \mathrm{MU}$ versus $555 \mathrm{MU}$, respectively). Studies that compare SmartArc-produced single-arc VMAT 

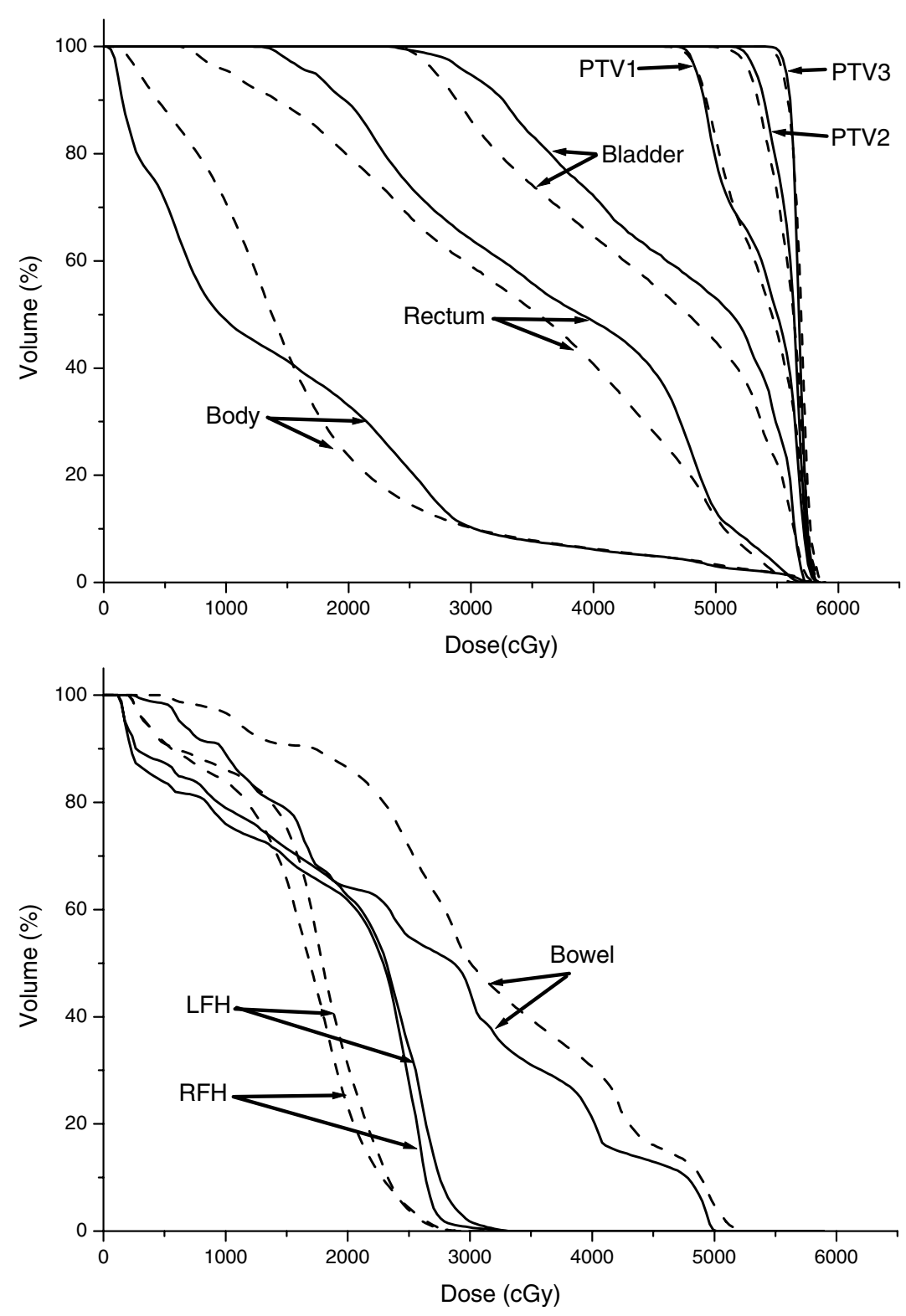

Figure 3. Dose-volume histogram (DVH) curves for one of the five prostate patients planned as part of the VMAT commissioning. The solid lines represent a standard IMRT approach, and the dashed lines represent the VMAT plan. LFH and RFH are the left and right femoral heads, respectively.

with step-and-shoot IMRT plans show a slightly larger reduction in monitor units $(\sim 10 \%$ reported by Bertelsen et al (2010), Guckenberger et al (2009)). Much larger differences in MU (up to 50\%) have been reported when comparing VMAT to sliding-window IMRT plans (Zhang et al 2009, Palma et al 2008), although this is mainly due to the nature of sliding-window delivery. 

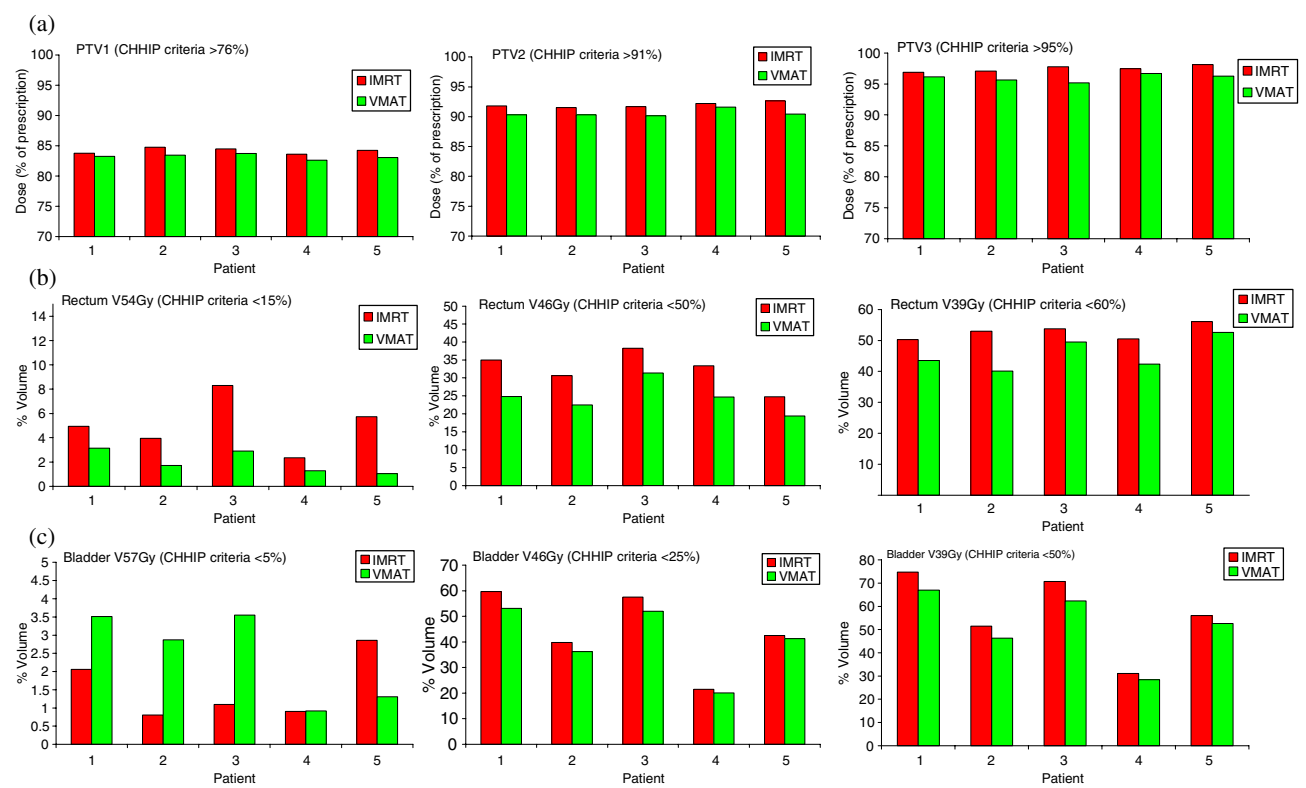

Figure 4. Comparison of CHHIP parameters for the IMRT and VMAT plans for the five commissioning patients. (a) Doses to the target volumes, which are similar between the two techniques. (b), (c) The volume of the rectum and bladder receiving three relevant CHHIP doses respectively (e.g. V54 Gy refers to the volume of the OAR receiving 54 Gy of the prescription dose).

\subsection{Verification}

All commissioned plans were transferred to the linear accelerator and delivered successfully. The mean delivery time was 2.5 min (range 2.3-2.9), and the clinical plans now being treated are of a similar duration. VMAT offers an improvement compared to the time taken to deliver five fixed IMRT fields, which for the commissioning patients examined here was on average $6.0 \mathrm{~min}$ (range 5.1-6.6).

All VMAT plans verified successfully on the Delta ${ }^{4}$ with $>95 \%$ of pixels within the $20 \%$ isodose having a gamma index of $<1$ at the $3 \% / 3 \mathrm{~mm}$ level. This is a similar level of verification achieved when using the Delta ${ }^{4}$ to verify IMRT prostate patients.

\subsection{Delivery resilience}

When using the original (uninterrupted) VMAT delivery as a reference on the Delta ${ }^{4}$, no significant deviation was observed for any of the resilience scenarios studied with a $100 \%$ pass for a gamma analysis of $2 \% / 2 \mathrm{~mm}$ within the $20 \%$ isodose being achieved in all cases. When a deliberate $5 \%$ asymmetry was introduced into the beam, the percentage gamma pass values remained at all times above $90 \%$ when compared to the reference plan delivered without any asymmetry.

\section{Discussion}

A VMAT planning solution has been developed using the Pinnacle v.8.0 treatment planning system supplemented by software developed in-house. Crucially, the VMAT solution 
demonstrated here can produce a multiple dose level plan capable of being delivered in a single arc in a shorter treatment time than IMRT. DVH analysis shows that the planning process produces target volume coverage of equivalent quality to this centre's IMRT solution for prostate patients. The VMAT solution also achieves lower doses for the OARs. When considering the CHHIP trial parameters, which provide a good overall indication of dosimetric quality, the VMAT plans again performed well.

The process of beginning with static beams that contain several step-and-shoot segments and sequencing them into an arc has been demonstrated elsewhere (Cao et al 2009) and is the starting point of the Pinnacle SmartArc optimization (Bzdusek et al 2009). The method described here also orders each pair of control points so that leaf motion is minimized. Using an in-house solution has enabled this department to investigate in detail the planning and delivery aspects of VMAT, and has allowed a greater degree of flexibility and control over the optimization.

In terms of efficiency, the VMAT plans demonstrate a delivery time similar to that reported elsewhere for single-arc prostate treatments treated with an Elekta linac (Bedford 2009, Cao et al 2009). Shorter treatment times have been reported for the Varian RapidArc solution ( $\sim 1$ min, Zhang et al (2009)), which is due in part to the availability of continuously variable dose rates for Varian linacs. Similarly, the modest reduction in monitor units required for VMAT plans compared to step-and-shoot IMRT plans is as expected. The literature suggests that SmartArc offers a potential $\sim 10 \%$ reduction in monitor units from step-and-shoot prostate IMRT, compared to $\sim 6 \%$ demonstrated here. Again, larger reductions have been reported comparing VMAT with sliding-window IMRT delivery.

The efficiency of VMAT delivery is strongly influenced by the planning strategy employed. While developing the prostate planning method outlined in this study, it was found that the speed and accuracy of VMAT delivery was improved when leaf motion outside of the treatment field was reduced. In practice, efforts can be made throughout the planning process to inspect the individual control points, identify any unnecessary leaf and jaw motion, and attempt to reduce it.

In summary, the VMAT planning solution demonstrated here delivers dose distributions of comparable quality to IMRT in a single arc and in a shorter treatment time. Delivery has been verified to a high degree of dosimetric accuracy and resilience tests also indicate that the solution is robust. This planning process has been introduced clinically for a subset of prostate patients at our institution.

\section{Acknowledgments}

The authors would like to thank Steve Smith for his assistance with the sequencing software, Dr Di Yan at the William Beaumont Hospital, Michigan, for help with the conversion into deliverable plans in MOSAIQ and the UK Elekta VMAT consortium.

\section{References}

Bedford J L 2009 Treatment planning for volumetric modulated arc therapy Med. Phys. 36 5128-38

Bedford J L, Lee Y K, Wai P, South C P and Warrington A P 2009 Evaluation of the Delta ${ }^{4}$ phantom for IMRT and VMAT verification Phys. Med. Biol. 54 N167-76

Bedford J L and Warrington A P 2009 Commissioning of volumetric modulated arc therapy (VMAT) Int. J. Radiat. Oncol. Biol. Phys. 73 537-45

Bertelsen A, Hansen C R, Johansen J and Brink C 2010 Single arc volumetric modulated arc therapy of head and neck cancer Radiother. Oncol. 95 142-8 
Bzdusek K, Friberger H, Eriksson K, Hårdemark B, Robinson D and Kaus M 2009 Development and evaluation of an efficient approach to volumetric arc therapy planning Med. Phys. 36 2328-39

Cao D, Afgan M K, Ye J, Chen F and Shepard D M 2009 A generalized inverse planning tool for volumetric-modulated arc therapy Phys. Med. Biol. 54 6725-38

Feygelman V, Zhang G and Stevens C 2010 Initial dosimetric evaluation of SmartArc-a novel treatment planning module implemented in a multi-vendor delivery chain J. Appl. Clin. Med. Phys. 283169

Guckenberger M, Richter A, Krieger T, Wilbert J, Baier K and Flentje M 2009 Is a single arc sufficient in volumetricmodulated arc therapy (VMAT) for complex-shaped target volumes? Radiother. Oncol. 93 259-65

Khoo V S and Dearnaley D P 2008 Question of dose, fractionation and technique: ingredients for testing hypofractionation in prostate cancer-the CHHiP trial Clin. Oncol. 20 12-4

Matuszak M M, Yan D, Grills I and Martinez A 2010 Clinical applications of volumetric modulated arc therapy Int. J. Radiat. Oncol. Biol. Phys. 77 608-16

Otto K 2008 Volumetric modulated arc therapy: IMRT in a single arc Med. Phys. 35 310-7

Palma D, Vollans E, James K, Nakano S, Moiseenko V, Shaffer R, McKenzie M, Morris J and Otto K 2008 Volumetric modulated arc therapy for delivery of prostate radiotherapy: comparison with intensity modulated radiotherapy and three-dimensional conformal therapy Int. J. Radiat. Oncol. Biol. Phys. 72 996-1001

Rao M, Yang W, Chen F, Sheng K, Ye J, Mehta V, Shepard D and Cao D 2010 Comparison of Elekta VMAT with helical tomotherapy and fixed field IMRT: plan quality, delivery efficiency and accuracy Med. Phys. 37 1350-9

Shaffer R, Nichol A M, Vollans E, Fong M, Nakano S, Moiseenko V, Schmuland M, Ma R, McKenzie M and Otto K 2010 A comparison of volumetric modulated arc therapy and conventional intensity-modulated radiotherapy for frontal and temporal high-grade gliomas Int. J. Radiat. Oncol. Biol. Phys. 76 1177-84

Webb S and McQuaid D 2009 Some considerations concerning volume-modulated arc therapy: a stepping stone towards a general theory Phys. Med. Biol. 54 4345-60

Zhang P, Happersett L, Hunt M, Jackson A, Zelefsky M and Mageras G 2009 Volumetric modulated arc therapy: planning and evaluation for prostate cancer cases Int. J. Radiat. Oncol. Biol. Phys. 76 1456-62 